\title{
Reminiscence and partial reinforcement in a discrete bar press situation'
}

On the day following varied number of discrete trials under $100 \%$ or $50 \%$ reinforcement in an operant conditioning cham. ber, rats were given nonre inforced trials until an extinction criterion of no bar press in $5 \mathrm{~min}$. was reached. An analysis of relative speed scores revealed significantly more reminisence for the $100 \%$ Ss. The data also indicated a reversal of the PRE when relative speed scores were analyzed and no significant difference between the $100 \%$ and $50 \%$ groups. when trials to criterion constituted the dependent variable.

In view of the large number of studies dealing with the effect of partial reinforcement upon extinction it is surprising that very little is known about the effect of reinforcement schedules upon such related phenomena as spontaneous recovery and reminiscence. A recent study by Thompson (1966) provided some additional information on spontaneous recovery and partial reinforcement. The present investigation was designed to secure data regarding reminiscence as a function of reinforcement schedules where the total number of acquisition trials as well as the number of reinforced trials could be equated.

Method

An operant conditioning chamber 10-1/2 in. high, $10-1 / 2$ in. wide, and 16 in. long was enclosed in a sound-shielded box and was controlled by programming equipment located $4 \mathrm{ft}$. from the box. The chamber was equipped with a Foringer retractable response bar located at one end and a food cup located at the other end into which $.045 \mathrm{gm}$ food pellets could be delivered. A floor switch located below the food cup released the bar 2 sec. following depression of the switch. Response time (total time between bar presses) was measured by two Hunter Silent Klockcounters, which were alternated at the end of each trial by a flop-flop switch. White noise was delivered through a speaker located behind the end of the chamber upon which the food cup was mounted.

The Ss, 80 naive male albino rats of the Holtzman strain 70 to 108 days old at the start of the experiment, were housed in individual living cages with ad-lib water. Following five days of $23 \mathrm{hr}$. food deprivation schedule each $\mathrm{S}$ was trained for two days by the method of approximation to press the bar and approach the food cup. On the third day Ss were given acquisition training and on Day 4 they were given nonreinforced trials until an extinction criterion of no bar press in 5 min. was reached.

The design included two levels of percentage reinforcement $(100 \%$ vs. $50 \%$ ) with the $100 \%$ Ss receiving $12,24,36,48$, or 72 acquisition trials and the $50 \%$ Ss receiving 24,48 , or 72 acquisition trials. The design may be schematized as two 2 by 3 factorials, in one of which the number of acquisition trials $(24,48,72)$ is equated for the two reinforcement conditions, while in the other the number of reinforced trials $(12,24,36)$ is equated. Ten Ss were randomly assigned to each of the eight experimental groups.

\section{Results and Discussion}

All time measures were converted to speed scores by a reciprocal transformation and the median of each block of five trials was determined. Since the performance levels at the end of acquisition differed significantly (the $50 \%$ Ss were faster) this data was then transformed to provide a measure of relative extinction with unity and zero based upon the reciprocals of the medians of each individual S's last five acquisition trials and last five extinction trials, respectively (see Anderson, 1963).

Two indices of reminiscence were employed: median relative speed for the first five extinction trials and relative speed of the first extinction trial. When the first of these measures was tested with the KruskalWallis $\mathrm{H}$ test, the $100 \%$ groups showed significantly more reminiscence, with $\mathrm{H}=18.59, \mathrm{df}=5, \mathrm{p}<.01$, when the groups were equated for total number of acquisition trials (see Fig. 1). The $100 \%$ groups were also superior (nonsignificant) when equated for number of reinforced

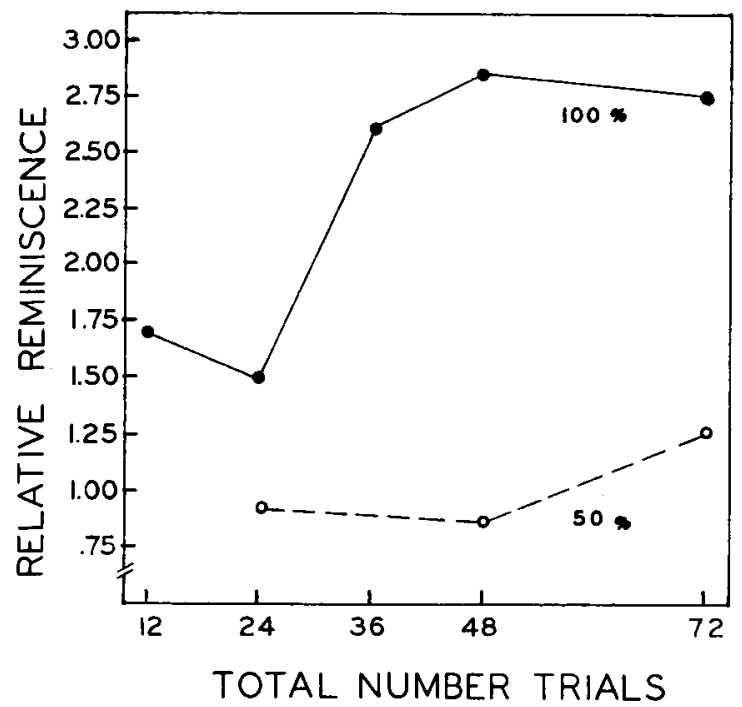

Fig. 1. Relative reminiscence (median median relative speed of first five extinction trials) as a function of number of acquisition trials and reinforcement condition. 
trials, $\mathrm{H}=7.63, \mathrm{df}=5$. Reminiscence scores based upon the relative speed of the first extinction trial also indicated that the $100 \%$ groups were significantly superior $(p<.05)$ when equated either for total number of acquisition trials or for number of reinforced trials, $H=11.11$ and 12.70 , $\mathrm{df}=5$, respectively .

Whether as a result of this differential amount of reminiscence, or the reduction of secondary reinforcement (by the elimination of multiple bar presses and the consequent forcing of a visit to the food cup on every trial), or both, the effect upon the PRE was pronounced. The $100 \%$ Ss showed significantly greater median relative speed for the first 25 extinction trials with $\mathrm{H}=\mathbf{2 1 . 7 6}$, $d f=5, p<.001$, when the two reinforcement schedule groups were equated for total number of acquisition trials, and $H=11.42$, df $=5, p<.01$, when equated for number of reinforced trials. A second measure of PRE, number of extinction trials to criterion, yielded no difference between the two reinforcement schedules. When subjected to a two-way analysis of variance test, neither the reinforcement condition variable nor the interaction was significant, although a significant trials effect was found, with $F=3.18$, df $=2 / 54, p<.05$, when the groups were equated for total number of trials, and $F=5.29, \mathrm{df}=2 / 54, \mathrm{p}<.01$, when the groups were equated for number of reinforced acquisition trials.

If, as suggested by the results of this study, the introduction of partial reinforcement results in a reduction of reminiscence, and if similarly, as indicated by Thompson (1966), partial reinforcement results in a reduction of spontaneous recovery, then studies of the PRE may be confounded when a $24 \mathrm{hr}$. time period elapses between acquisition and extinction, or when extinction is not completed in one session.

\section{References}

Anderson, N. H. Comparison of different populations: resistance to extinction and transfer. Psychol. Rev., 1963, 70, 162-179.

Thompson, M. E. Spontaneous recovery and partial reinforcement effects in the runway. Psychon. Sci., 1966, 5, 33-34.

\section{Note}

1. This investigation was supported by a research grant, G-19274, from the National Science Foundation. The data were collected by Paul Hettich and I-Ning Huang. Appreciation is expressed to Jean P. Thompson for valuable assistance in preparaing this paper. 